\title{
Erratum to: The distinct roles of anion transporters SIc26a3 (DRA) and Slc26a6 (PAT-1) in fluid and electrolyte absorption in the murine small intestine
}

\author{
Weiliang Xia • Qin Yu • Brigitte Riederer • Anurag Kumar Singh • \\ Regina Engelhardt • Sunil Yeruva • Penghong Song • De-An Tian • \\ Manoocher Soleimani • Ursula Seidler
}

Published online: 16 April 2014

(C) Springer-Verlag Berlin Heidelberg 2014

\section{Erratum to: Pflugers Arch - Eur J Physiol}

DOI 10.1007/s00424-013-1381-2

The original version of this article inadvertently contained a mistake.

The correct Author contributions is provided here:

Author contributions

W.X., Q.Y., B.R., A.K.S., R.E., and U.S. designed, performed and analysed experiments, S.Y., P.S., D.A.T., and M.S. provided expert assistance and suggestions, and W.X:, Q.Y., B.R. and U.S. wrote the manuscript.

The online version of the original article can be found at http://dx.doi.org/ 10.1007/s00424-013-1381-2.

W. Xia $\cdot$ Q. Yu $\cdot$ B. Riederer · A. K. Singh $\cdot$ R. Engelhardt •

$\mathrm{S}$. Yeruva $\cdot$ U. Seidler

Department of Gastroenterology, Hannover Medical School,

Hannover, Germany

Q. Yu - D.-A. Tian

Department of Gastroenterology, Tongji Hospital, Huazhong

University of Science and Technology, Wuhan, China

P. Song

Key Laboratory of Combined Multiorgan Transplantation, The First

Affiliated Hospital, School of Medicine, Zhejiang University,

Hangzhou 310003, China

M. Soleimani

Center on Genetics of Transport and Epithelial Biology,

University of Cincinnati, Cincinnati, OH, USA

U. Seidler $(\bowtie)$

Department of Gastroenterology, Hepatology and Endocrinology,

Hannover Medical School, Carl-Neuberg-Straße 1,

0625 Hannover, Germany

e-mail: seidler.ursula@mh-hannover.de 